\title{
Synthesis and Characterization of L-Alanine Sodium Bromide: A New Semi Organic Nonlinear Optical Material
}

\author{
T. Jayanalina \\ Department of Physics, \\ KSR College of Engineering, \\ Tiruchengode-637215, \\ Tamil Nadu, India.
}

\author{
G. Rajarajan \\ Department of Physics, \\ Mahendra Engineering College, \\ Mallasamudaram, Namakkal, \\ Tamil Nadu, India
}

\begin{abstract}
A new semi organic nonlinear optical crystal, L-Alanine Sodium Bromide has been grown from aqueous solution by slow evaporation technique. L-Alanine and Sodium Bromide were used in the ratio 1:1 for synthesis. Crystals of size $20 \times 10 \times 4 \mathrm{~mm}^{3}$ have been obtained in 30 days. Characterizations were carried out to study the structural, optical and mechanical properties of the grown crystals. Single crystal X- ray diffraction analysis shows that the grown crystal belongs to the monoclinic system. The crystalline nature and its various planes of reflections were observed by the powder XRD analysis. The chemical compositions of the grown crystals were determined by FTIR spectral analysis. The transmittance, absorption and reflection of the crystal were studied by UV- Visible spectrometer. The mechanical properties of the grown crystals were studied using Vickers micro hardness measurement.
\end{abstract}

Keywords: Characterization, Slow evaporation, Crystal structure, Nonlinear material.

\section{INTRODUCTION}

The search for efficient and new materials on nonlinear optical process has been very active since the discovery of second harmonic generation (SHG) in quartz crystal by Franken et al [1]. Nonlinear optical [NLO] materials are expected to play a major role in photonics including optical information processing, telecommunication sensor protector applications, optical data storage, etc. Some organic compounds exhibit large NLO response in many cases, order of magnitude larger than widely known inorganic materials. They also offer the flexibility of molecular design and the promise of virtually an unlimited number of crystalline structures. In this stimulating context, organic nonlinear materials have been recognized as forefront candidate for fundamental and applied investigations involving, in a joint effort, chemists, material scientists and optical engineering [2,6 ]. Over past two decades, there has been remarkable interest in growth and characterization of nonlinear optical material crystals [5, 8]. Second order nonlinear optical materials are used in optical switching, frequency conversion and electro-optical applications especially in Electro optical modulators [9, 10]. In addition to large second order susceptibilities, good transmission in UV and visible region and stable physio-thermal performance are needed for these applications [11, 12]. Inorganic NLO materials have large mechanical strength, thermal stability and good transmittance but modest optical nonlinearity due to the lack of extended $\pi-$ electron dislocation $[13,15]$. Purely organic NLO material have large nonlinearity compared to inorganic material but low optical transparency, poor mechanical and thermal strength and low laser damage threshold. Thus the research is focused on semi- organic NLO material crystal in order to obtain superior NLO crystal by combining the advantages of organic and inorganic materials. The semi-organic NLO materials have been attracting much attention due to high nonlinearity, chemical flexibility, high mechanical and thermal stability and good transmittance [16]. Most of the amino acids individually exhibit the NLO property due to donor amino group $\mathrm{NH}^{3+}$ and acceptor carboxyl group $\mathrm{COO}^{-}$and intermolecular charge transfer is also possible [17]. Semi-organic nonlinear optical [NLO] crystals are formed by amino acids with inorganic materials possess the advantages of high optical nonlinearity of the organic amino acids [18]. L- Alanine is the simplest amino acid having SHG efficiency one third of that of the well known KDP, but the knowledge of studying its properties is very important since LAlanine can be considered as the fundamental building block of more complex amino acids [19]. L-Alanine mixed semi organic material will be special interest as a fundamental building block to NLO properties [20]. Presently, we are interested to study the growth, structural, optical and hardness studies of L-Alanine Sodium Bromide by slow evaporation technique. Also the grown crystals have subjected to various characterizations such as Single crystal X- ray diffraction, Powder XRD, FTIR, UVVisible spectroscopy, and Micro hardness studies.

\section{EXPERIMENTAL DETAILS}

\subsection{Crystal Growth}

L- Alanine and Sodium Bromide obtained from E. Merck [India] Ltd were mixed in a stoichiometric ratio of 1:1 in triple distilled water and stirred continuously for six hours for homogenization. The synthesized substance was purified by the repeated recrystillization process. A saturated growth solution was prepared and kept at room temperature for slow evaporation. Good quality single crystal with regular shape and size $20 \times 10 \times 4$ $\mathrm{mm}^{3}$ was harvested within 30 days as shown in Figure.1. 


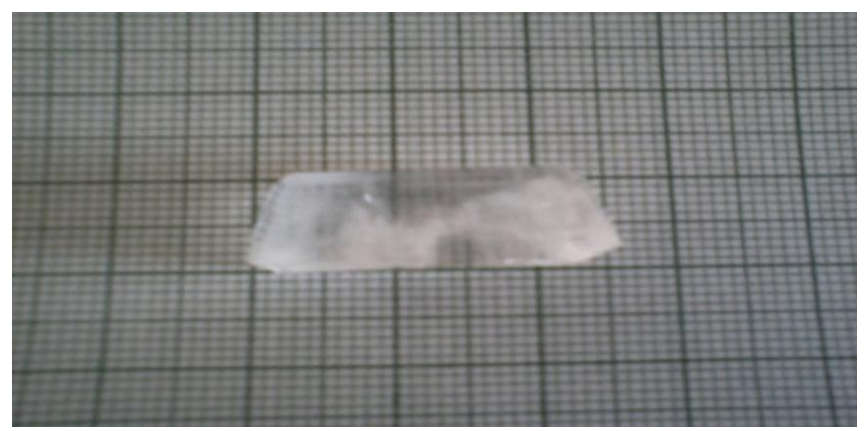

Figure. 1. Photograph of L-ANaBr Crystal

\section{RESULTS AND DISCUSSION}

\subsection{Single crystal $X$ - ray diffraction analysis}

The single $\mathrm{X}$ - ray diffraction studies have been carried out to confirm the crystalline nature and to determine the lattice parameters of the grown crystal. The single crystal X- ray diffraction has been carried out using an ENRAF NONIUS FR590 diffractometer with $\mathrm{M}_{\mathrm{o}}$ radiation of wavelength $\lambda=0.71073 \mathrm{~A}^{\circ}$ and the lattice parameter values are presented in Table 1.

Table 1: Lattice parameter values of the grown L-ANaBr Crystal.

\begin{tabular}{|c|c|c|c|c|c|c|}
\hline Crystal & $\mathrm{a}\left(\mathrm{A}^{\mathrm{o}}\right)$ & $\mathrm{b}\left(\mathrm{A}^{\mathrm{o}}\right)$ & $\mathrm{c}\left(\mathrm{A}^{\mathrm{o}}\right)$ & $\mathrm{V}^{\mathrm{o}} \mathrm{A}^{3}$ & $\alpha=\beta=\gamma$ & System \\
\hline $\begin{array}{c}\text { L- Alanine } \\
\text { Sodium } \\
\text { Bromide }\end{array}$ & 6.03 & 5.80 & 12.38 & 433 & $90^{\theta}$ & Mono Clinic \\
\hline
\end{tabular}

\subsection{Powder X-ray diffraction analysis}

The grown crystals have been characterized by powder X- ray diffractometer to verify the single phase nature of the sample. Well defined Bragg peaks are obtained at specific $2 \theta$ angles indicating that crystals are ordered. The peaks in the Figure 2 show the crystalline nature of $\mathrm{L}-\mathrm{ANaBr}$. The crystalline size (D) were calculated using the Scherrer's formula from the full width half maximum $(\beta)$ using the relation.

$$
\mathrm{D}=\frac{0.945 \lambda}{\beta \cos \theta}
$$

The crystalline size of the grown crystal was found to be $59 \mathrm{~nm}$.

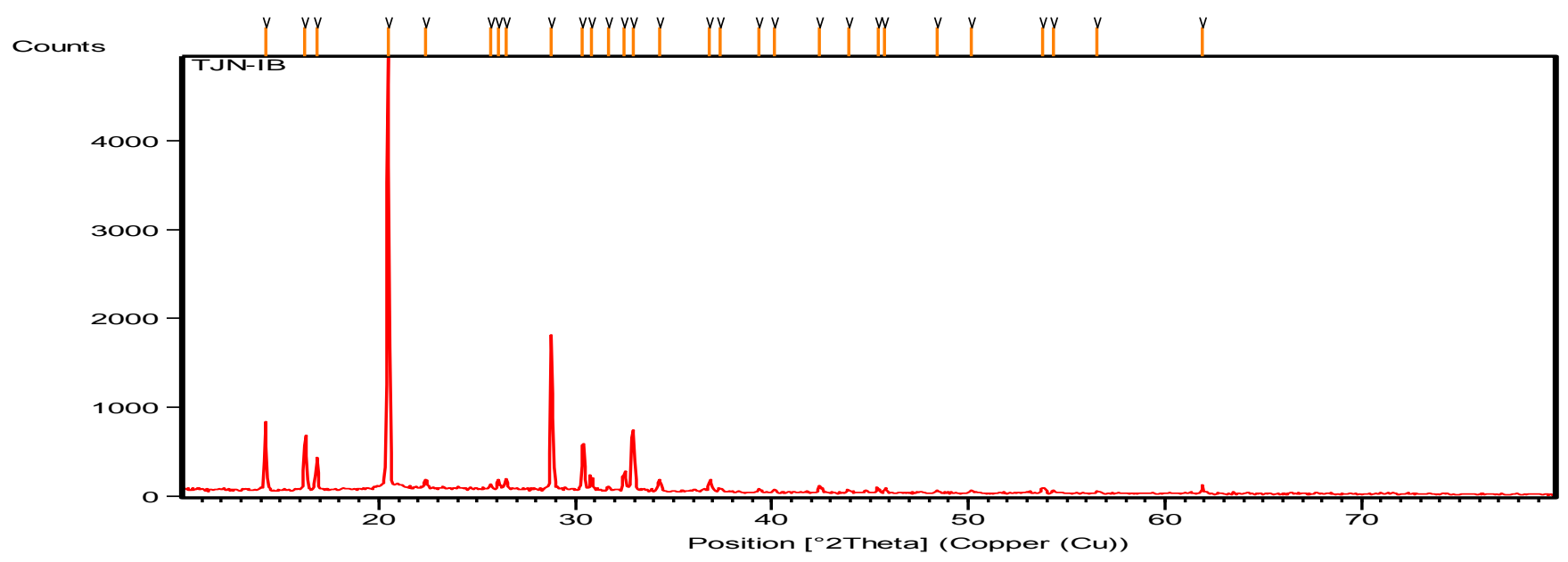

Figure. 2. Powder X-ray diffraction analysis of $\mathrm{L}-\mathrm{ANaBr}$

\subsection{FT- IR Spectroscopic analysis}

The FTIR analysis was carried out using Alpha Bruker FTIR spectrometer by $\mathrm{KBr}$ pellet technique in the range $500-4000 \mathrm{~cm}^{-}$ ${ }^{1}$ to confirm the presence of different organic groups with the inorganic materials present in the Table 2. The FTIR spectrum of the grown crystal is shown Figure 3. The presence of carboxyl group around $3000 \mathrm{~cm}^{-1}$ can be observed due to Alanine presence. The internal vibrations of Alanine are observed on the functional groups $\left[\mathrm{NH}_{3}^{+}, \mathrm{CH}_{2}, \mathrm{COO}^{-}\right.$] which is in agreement with the data reported before [ Lydia et al. , 2009 ]. $3086 \mathrm{~cm}^{-1}$ with medium intensity represents $\mathrm{C}-\mathrm{H}$ asymmetric stretching. $2937 \mathrm{~cm}^{-1}$ with weak intensity shows $\mathrm{OH}$ stretching. The peak at $1620 \mathrm{~cm}^{-1}$ with strong intensity represents $\mathrm{C}=\mathrm{O}$ stretching. The peak at $2292 \mathrm{~cm}^{-1}$ with medium intensity represents overtones $\&$ combination bands with prominent peaks near 2500 and $2000 \mathrm{~cm}^{-1}$. The peaks at $1014 \mathrm{~cm}^{-1}$ with variable represent $\mathrm{C}-\mathrm{CHO}$ stretching. The peak at $1455 \mathrm{~cm}^{-1}$ with medium intensity represents $\mathrm{C}-\mathrm{H}$ in plane bending. 


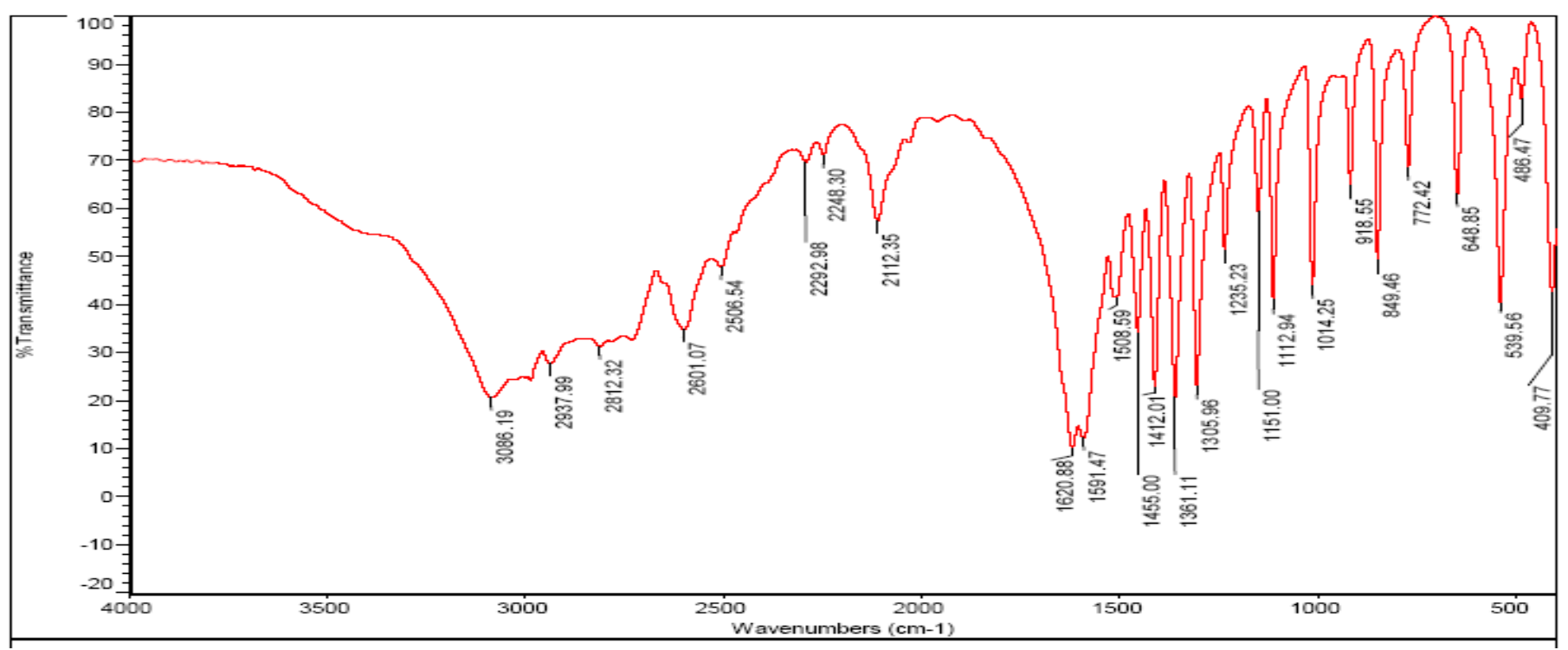

Figure. 3. FTIR Spectra of L-ANaBr

Table. 2: Characteristic Absorption Frequencies of Various Functional Groups

\begin{tabular}{|c|c|c|c|}
\hline S.No & Frequency Range & Intensity & Mode of vibration \\
\hline 1. & 3086 & $\mathrm{~m}$ & $\mathrm{C}-\mathrm{H}$ asym. stretching \\
\hline 2. & 2937 & w (broad) & OH stretching \\
\hline 3. & 2601 & w (broad) & OH stretching \\
\hline 4. & 2506 & w (broad) & OH stretching \\
\hline 5. & 2292 & $\mathrm{~m}$ & $\begin{array}{l}\text { Overtones \& combination bands with prominent peaks near } 2500 \text { and } \\
2000 \mathrm{~cm}^{-1}\end{array}$ \\
\hline 6. & 2248 & $\mathrm{~m}$ & $\begin{array}{l}\text { Overtones \& combination bands with prominent peaks near } 2500 \text { and } \\
2000 \mathrm{~cm}^{-1}\end{array}$ \\
\hline 7. & 2112 & $\mathrm{~m}$ & $\begin{array}{l}\text { Overtones \& combination bands with prominent peaks near } 2500 \text { and } \\
2000 \mathrm{~cm}^{-1}\end{array}$ \\
\hline 8. & 1620 & $\mathrm{~s}$ & $\mathrm{C}=\mathrm{O}$ stretching \\
\hline 9. & 1591 & $\mathrm{~s}$ & $\mathrm{C}=\mathrm{O}$ stretching \\
\hline 10. & 1508 & $\mathrm{~s}$ & $\mathrm{NH}^{+3}$ sym. bending \\
\hline 11. & 1455 & $\mathrm{~m}$ & $\mathrm{C}-\mathrm{H}$ in plane bending \\
\hline 12. & 1412 & $\mathrm{w}$ & $\mathrm{C}-\mathrm{H}$ in bending (in plane) \\
\hline 13. & 1361 & $\mathrm{~W}$ & $\mathrm{C}-\mathrm{H}$ in bending (in plane) \\
\hline 14. & 1305 & $\mathrm{~W}$ & $\mathrm{C}-\mathrm{H}$ in bending (in plane) \\
\hline 15. & 1235 & $\mathrm{~s}$ & $\mathrm{C}-\mathrm{O}-\mathrm{C}$ stretching \\
\hline 16. & 1151 & $\mathrm{~s}$ & $\mathrm{C}-\mathrm{O}-\mathrm{C}$ stretching \\
\hline 17. & 1112 & $\mathrm{~m}$ & Sym.C $-\mathrm{O}-\mathrm{C}$ stretching \\
\hline 18. & 1014 & variable & $\mathrm{C}-\mathrm{CHO}$ stretching \\
\hline 19. & 918 & $\mathrm{w}$ & $\mathrm{C}-\mathrm{H}$ out of plane bending \\
\hline 20. & 849 & $\mathrm{~W}$ & C- H out of plane bending \\
\hline 21. & 772 & $\mathrm{~m}$ & $\mathrm{~N}-\mathrm{H}$ out of plane bending \\
\hline 22. & 648 & $\mathrm{~m}$ & OCN deformation \\
\hline 23. & 539 & $\mathrm{~s}$ & OCN deformation \\
\hline
\end{tabular}




\subsection{Optical transmission studies}

The UV-VIS spectrum analysis has been observed using Perkin Elmer Lambda 35 UV- Visible spectrophotometer. The UV-VIS spectrum gives limited information about the structure of the molecule because of the absorption of UV and visible light involves promotion of the electron in $\sigma$ and $\pi$ orbital form the ground state to higher energy states. To find the transmission, absorption and reflection range of newly formed $\mathrm{L}-\mathrm{ANaBr}$, the optical transmission spectrum of $\mathrm{L}-\mathrm{ANaBr}$ for the wavelengths between 500 and $2000 \mathrm{~nm}$ was recorded. The recorded optical transmittance, absorption and reflection spectrum are shown in Figures 4, 5, 6.
The transmittance is found to be maximum in the entire visible and infrared regions. The crystal shows a good transmittance in the entire visible regions. The lower cutoff $190 \mathrm{~nm}$ combined with the above, attest the useful of this material for optoelectronics application and the second harmonic generation of the Nd:YAG laser and for the generation of the higher harmonic of the laser diodes. A good optical transmittance is very desirable in a nonlinear optical [NLO] crystal since the absorptions, if any, in an NLO material near the fundamental or the second harmonic of an Nd: YAG laser will lead to loss conversion efficiency of second harmonic generation [SHG]. The crystal is highly transparent to the wavelengths above $190 \mathrm{~nm}$ to $1500 \mathrm{~nm}$. [21].

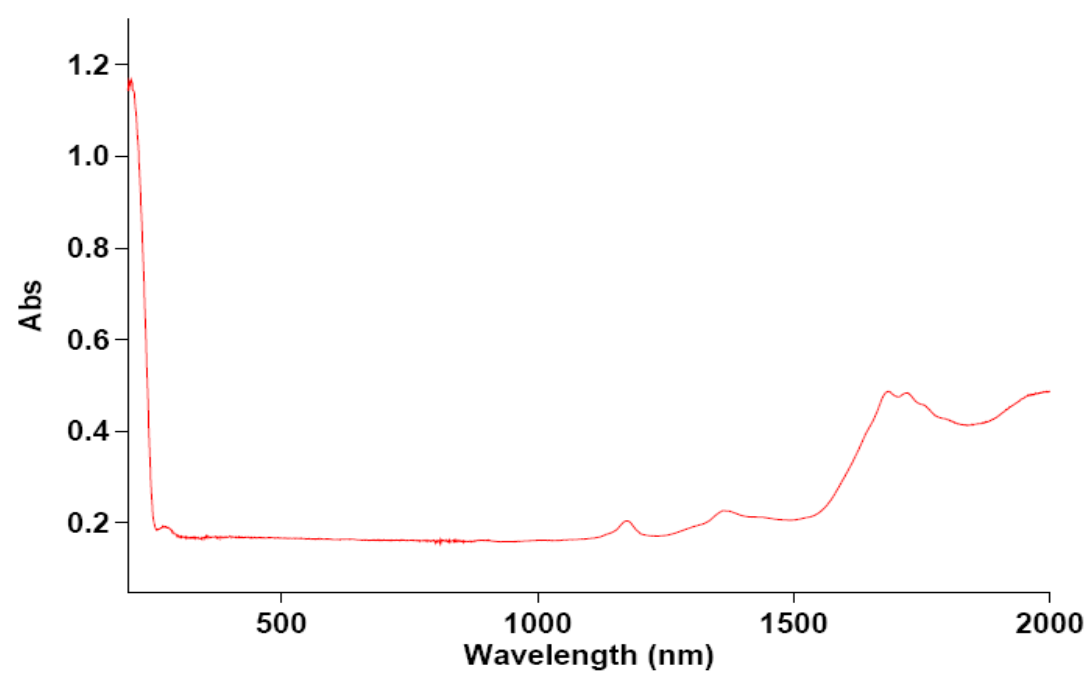

Figure. 4. UV-Visible absorption spectra of $\mathrm{L}-\mathrm{ANaBr}$

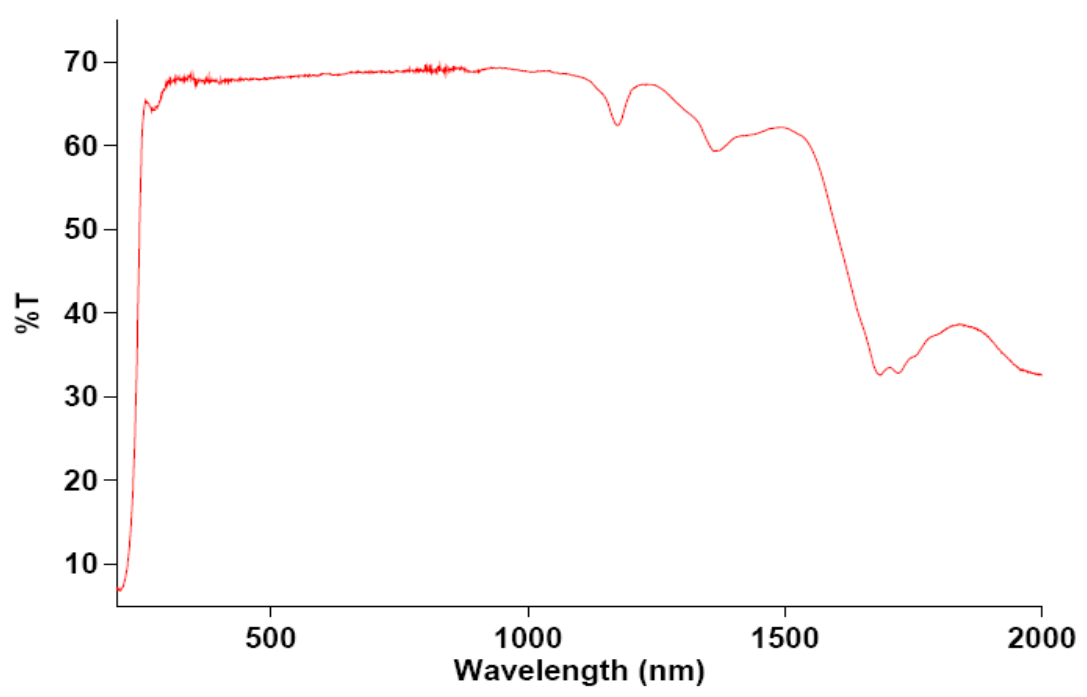

Figure. 5. UV-Visible transmission spectra of L-ANaBr 


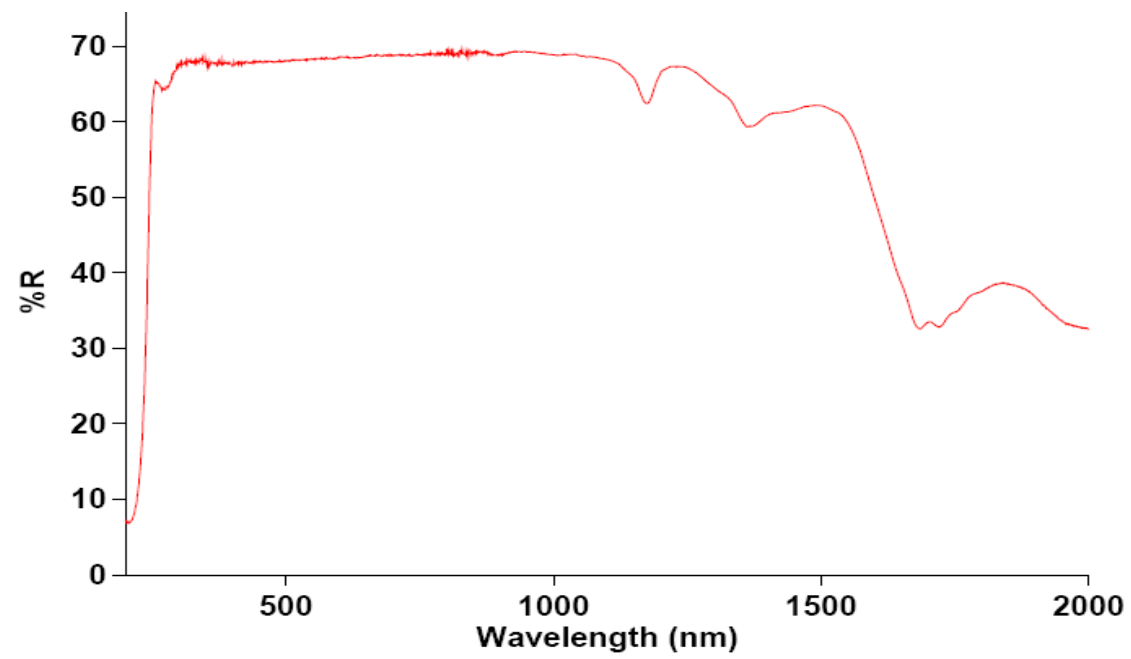

Figure. 6. UV-Visible reflection spectra of $\mathrm{L}-\mathrm{ANaBr}$

\subsection{Micro hardness measurement}

The definition of hardness depends entirely on the method of measurement which will determine the scale of hardness obtained. Hardness of a material is a measure of its resistance it offers to local deformation. An important use of micro hardness study is the possibility of making an indirect estimate of other mechanical characteristics of materials having a specific correction with their hardness. It plays a key role in device fabrication. Transparent crystals free from cracks were selected for micro hardness measurement. L-ANaBr crystal was subjected to Vickers micro hardness test with the load varying from 25 to $100 \mathrm{~g}$. Hardness number of the crystal was calculated using the following relation,

$$
\mathrm{H}_{\alpha}=\frac{1.8544 P}{d^{2}} \mathrm{Kg} / \mathrm{mm}^{2}
$$

Vickers micro hardness profile as a function of the applied test loads is illustrated in Figure 7. It is evident from the plot that the micro hardness of the crystal with load increases with increasing the load. The value of the work hardening co-efficient ' $n$ ' was found to be 0.08 . According to Onitsch, $1.0 \leq n \leq 1.032$ for hard material and $n>1.032$ for soft material [22]. Hence it is concluded that $\mathrm{L}-\mathrm{ANaBr}$ belongs to the soft material.

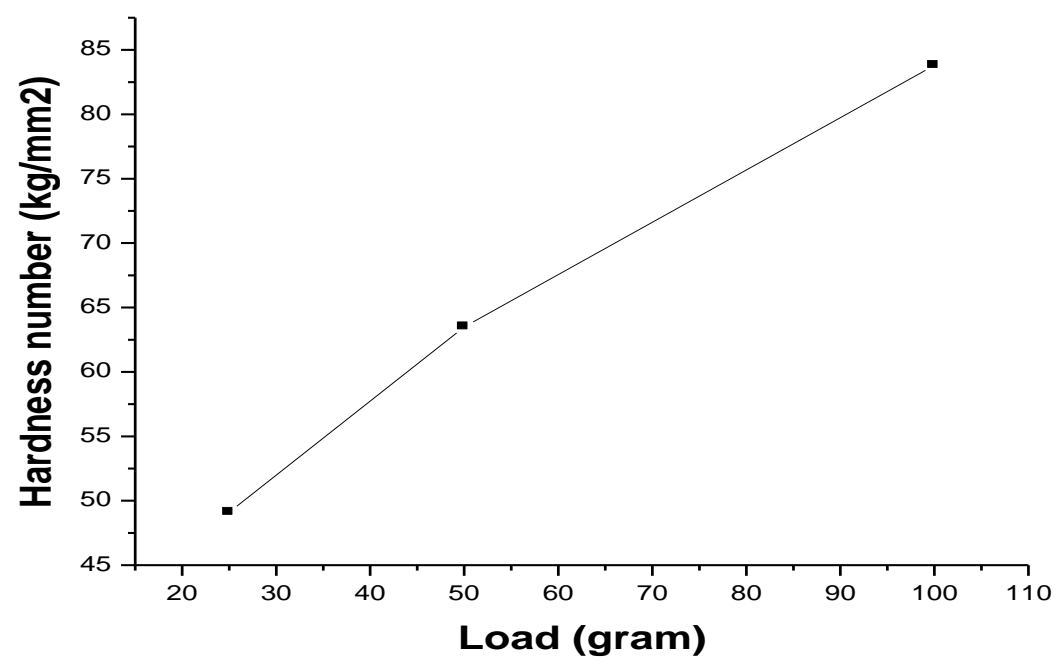

Figure. 7. Variation of micro hardness with load of $\mathrm{L}-\mathrm{ANaBr}$ 


\section{CONCLUSION}

Single crystals of L-Alanine Sodium Bromide [L-ANaBr] have been grown from aqueous solution by slow evaporation technique. Single crystal XRD reveals the structure of the crystal belongs to monoclinic system. The lattice parameters have been found by single crystal X-ray diffraction technique. The grown crystals were characterized by using powder XRD diffraction and it reveals the crystalline nature of the crystal. The FT-IR spectrum of the grown crystal reveals that the various functional groups present in the grown crystal. The optical absorption spectral studies confirm that the crystals have very good transmission in entire visible region, with lower cut-off around $190 \mathrm{~nm}$, which is an essential consideration for NLO crystals and it can also possibly be used for the fabrication of electro-optic devices. The Vickers micro hardness was calculated in order to understand the mechanical stability of the grown crystals.

\section{REFERENCES}

[1] P.A.Franken, A.E.Hill , C.W.Petess , Phys.Rev.Lett. 71, $18(1961)$

[2] S.Meenakshisundaram,S.Parthiban,G.Bhagavannarayana, G.Madhurambal, S.C.Mojumdar,J.Therm.Anal.Calorim.9, 125 (2009).

[3] M.D.Shirsat,S.S.Hussaini,N.R.Dhumane, V.G.Dongre, Crys t.Res.Technol.43,756 (2008).

[4] S.S.Hussaini , N.R.Dhumane, V.G.Dongre, P.Karmuse , P.Ghughare , M.D.Shirsat , J.Optelectron.Adv.Mater.Rapid Commun. 2, 108 (2008).

[5] K.Meera , R.Muralidharan , R.Dhanasekaren , ManyumPrapun , P.Ramasamy, J.Cryst.Growth. 263 , 510 (2004).

[6] G.D.Andreeti , L.Cavalca , A.Musatti , Acta Crystallogr. Sect. B 24, 683 (1968).

[7] R.MohanKumar, D.RajanBabu, D.Jayaraman , R.Jayavel, K.Kitamura, J. Cryst.Growth . 275, 1935 (2005).
[8] A.S.HajaHameed , C.W.Lan, J. Cryst. Growth. 270, 475 (2004).

[9] S.S.Hussaini , N.R.Dhumane ,V.G.Dongre , M.D.Shirsat , J.Materials Science-Poland, 27, 365 (2009).

[10] ShaokangGao, Weijun Chen, Guimei Wang, Jianzhong Chen, J. Cryst.Growth. 297,361 (2006).

[11] P.Joseph Ginson, J.Philip , K.Rajarajan , S.A.Rajasekar , A.Joseph Arul Pragasam , K.Thamizharasan , S.M. Ravi Kumar, P. Sagayaraj, J. Cryst.Growth. 296, 51 (2006).

[12] P.M.Ushasree, R.Muralidharan, R.Jayavel, P.Ramasamy, J.Cryst.Growth. 210, 741,(2000).

[13] H.Q.Sun, D.R.Yuan, X.Q. Wang, X.F.Cheng, C.R. Gong, M.Zhou, H.Y.Xu, X.C.Wei, C.N Luan, D.Y. Pan, Z.F.Li, X.Z. Shi, Cryst.Res.Technol. 40, 882 (2005).

[14] Ramajothi,S.Dhanuskodi,K.Nagarajan, Cryst.Res.Technol. 39, 414 (2004).

[15] S.Ariponnammal,S.Radhika, R.Selva , N.VictorJeya , Cryst.Res.Technol . 40, 786, (2005).

[16] Min-hua Jiang, Qi Fang, Adv. Mater. 11, 1147 (1999).

[17] P.M.Ushasree,R.Jayavel,P.Ramasamy, Mater.Chem. Phys. 61, 270 (1999).

[18] N.R. Dhumane, S.S.Hussaini, Kunal Datta, Prasanta Ghosh, Mahendra D.Shirsat,Rec. Res. in Sci. and Technol. 2(10), 30 (2010).

[19] Misoguti, L: Varela, A.T.; Nunes, F.D ; Bagnato, V.S.; Mela, F.E.A.; Mendes Filho.; Zilio, S.C. Opt.Mater, 6, 147152 (1996).

[20] M.Chitra, S.Palaniswamy, Rasayan J. Chem., 3(1), 145150 (2010).

[21] K.Kanagasabapathy, R.Rajasekaran, J.Optelectron.Adv.Mater.Rapid Commun. 6,218 (2012).

[22] E.M.Onitsch, Mikroskopie, 95, 12 (1998). 